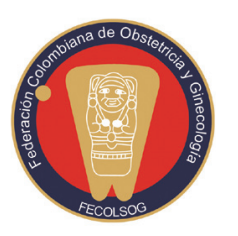

CARTAS al editor

\title{
COMENTARIO SOBRE EL ARTÍCULO "ANÁLISIS DE COSTO-EFECTIVIDAD DEL USO DE CALCIO MÁS ÁCIDO LINOLEICO PARA LA PREVENCIÓN DE LA HIPERTENSIÓN INDUCIDA POR EL EMBARAZO EN MUJERES CON RIESGO EN COLOMBIA"
}

\author{
Comments on "Cost-effectiveness analysis of \\ using calcium plus conjugated linolenic acid for \\ preventing pregnancy-induced hypertension in \\ females at risk in Colombia"
}

Medellín, 28 de noviembre de 2012

\section{Doctor Hernando Gaitán \\ Editor \\ Revista Colombiana de Obstetricia y Ginecología}

En la edición de la Revista Colombiana de Obstetricia y Ginecología, vol. 63, No. 3 (julio-septiembre de 2012) salió publicado el artículo de Martín Romero-Prada et al., titulado "Análisis de costo-efectividad del uso de calcio más ácido linoleico para la prevención de la hipertensión inducida por el embarazo en mujeres con riesgo en Colombia", el cual me llamó la atención por el tipo de análisis económico en salud que presenta, que es poco frecuente en la revista, y por referirse a un problema de salud pública en Colombia, tal como son los trastornos hipertensivos del embarazo. El artículo presenta en mi opinión algunas ligerezas que vale la pena aclarar:

A lo largo del manuscrito científico se presentan inconsistencias con el término hipertensión inducida por el embarazo (HIE), que no es utilizado en la literatura científica desde finales de los años noventa debido a que ocasiona confusión, pues se engloban en una misma enfermedad diferentes patologías, con distinta etiología y disímil pronóstico materno y perinatal. Los autores, en la introducción del artículo, referencian este término como el sugerido por el "Report of the National High Blood Pressure Education Program Working Group on High Blood Pressure in Pregnancy" (1), sin embargo, en este consenso norteamericano, contrario a lo expresado por los autores, lo que se intentó fue evitar el término HIE y tratar de involucrar y categorizar los trastornos hipertensivos durante la gestación. La etiología de la preeclampsia temprana o tardía, la hipertensión gestacional, la eclampsia y la hipertensión arterial crónica comparten algunas características, pero los fenómenos etiológicos, la progresión de la enfermedad y la severidad son diferentes, y hoy por hoy plantear una investigación, así sea desde el punto de vista económico, sin tener claramente definido el problema, plantea dificultades metodológicas y conclusiones erróneas, como al final lo señalan los autores.

La sustentación bibliográfica en la cual se fundamenta el artículo científico para proponer el uso del ácido linoleico más calcio en la llamada "HIE" data de la década de los noventa basados en unas observaciones clínicas y experimentales de Herrera et al., y en dos pequeños estudios aleatorizados con 
escaso número de pacientes realizado en Bangladesh y Colombia $(2,3,4)$. Estos hallazgos publicados por el doctor Herrera et al. no han sido replicados en la literatura colombiana o mundial; a pesar de esto, son sugeridos por el Ministerio de la Proteccion Social de Colombia para la prevención de la preeclampsia en pacientes con riesgo; sin embargo, esta práctica clínica carece de validación científica mundial y es objeto de debate. Realizar un análisis económico como el que plantean los autores, basado solamente en información dictada por pequeños artículos, es arriesgado, particularmente cuando no ha sido replicado en otras latitudes y no es una práctica sugerida basada en un nivel de evidencia científica con peso estadístico.

El autor principal del estudio, quien además dirige una fundación de estudios económicos en salud (www.salutia.org), teniendo en cuenta las pequeñas investigaciones realizadas en centros ambulatorios de Bangladesh y Colombia, erróneamente concluye que la mortalidad materna y la eclampsia, y la mortalidad perinatal son mayores en el grupo control, lo cual no fue expresado por Herrera et al. en su artículo original, quienes simplemente se limitaron a decir que, en su estudio, la presencia de trastornos hipertensivos fue notablemente menor en el grupo de manejo activo con calcio más ácido linoleico que en el de placebo. Se debe aclarar que la edad gestacional al parto y las tasas de cesárea no difirieron de manera significativa entre los dos grupos de estudio.

Los resultados y la metodología del estudio presentado por Romero-Prada et al. son bastante complejos y poco reproducibles. En la sección de materiales y métodos del análisis económico, además del posible evento muerte materna o perinatal, analizan supuestas tasas de partos, cesáreas y prematuridad basados en estudios clínicos de poco peso estadístico y en poblaciones diferentes a la colombiana $(6,7,8)$, por tanto, en mi opinión, estos resultados no tienen una adecuada confiabilidad como para hacer el análisis económico planteado en nuestra población.

En los resultados, los autores concluyen: "Aunque el caso base se hizo teniendo en cuenta una cohorte de solo 100 mujeres en riesgo, es importante recordar que la incidencia de la HIE en Colombia es superior al 8\% y se reportan 699.775 nacidos vivos para el 2009, por lo que el impacto sería mucho mayor en términos de las vidas salvadas con un costo que podría significar menos del 0,05\% de la UPC si se diera a todas las mujeres en embarazo cubiertas por el sistema de seguridad social". Es decir, los autores del estudio económico van más allá de las observaciones clínicas realizadas en el pasado por Herrera et al., quienes utilizaron la combinación del ácido linoleico más calcio en pacientes con "alto riesgo de preeclampsia", y en ningún momento para toda la población de gestantes colombianas. Este planteamiento universal en las gestantes no tiene ningún respaldo científico que lo avale.

Por último, y no menos importante, los autores del artículo expresan: "Conflicto de intereses: ninguno declarado. Financiación: Tecnoquímicas realizó una donación que permitió financiar parte de esta investigación”. Esta aclaración es, en mi opinión corta, pues la fundación de estudios económicos en salud que dirige el doctor Romero-Prada (autor principal del artículo) recibió financiación directa para la elaboración del artículo en mención, pues en su página web ${ }^{1}$ mencionan que Tecnoquímicas fue la entidad "contratante". Creo que bajo los pretextos de conflicto de intereses en investigación clínica, en este análisis económico publicado existe un claro conflicto de intereses. Según Camí (9): "Se entiende por conflicto de intereses aquella situación en la que el juicio de un profesional concerniente a su interés primario (p. ej., la salud de los enfermos o la integridad de una investigación) tiende a estar indebidamente influenciado por un interés secundario (de tipo económico o personal). Con independencia de que el interés secundario sea necesario o deseable, se produce un conflicto cuando este prevalece sobre el interés primario. Los conflictos de intereses no son directamente el resultado de unos comportamientos, sino unas situaciones en las que se dan determinadas circunstancias". Por tanto, considero que si la entidad contratante (Tecnoquímicas) acordó los servicios de

http:/www.salutia.org/2011-09-08-14-48-20/noticias/78-lineas-deinvestigacion/88-investigaciones-realizadas-2009-2011 
investigación en salud de Romero-Prada et al., existe un claro conflicto de intereses económicos, lo cual no es deplorable, por el contrario, es interesante, pues la alianza con la industria farmacéutica permite el avance de la ciencia, sin embargo creo que, voluntaria o involuntariamente, los autores omitieron esta información. El doctor Romero-Prada y su grupo han realizado este tipo de estudios económicos para diferentes empresas de la industria farmacéutica (Bayer, Pfizer, Roche, Novartis, Aztra Seneca, entre otros $)^{2}$, lo cual puede ser justificado para introducir medicamentos y para analizar su costo-efectividad; no obstante, no es para mí claro si el investigador y su grupo avalarán y publicarán los resultados de un estudio económico contratado si son desfavorables para la empresa farmacéutica).

Reconozco el esfuerzo y la trayectoria científica de los autores del artículo en la elaboración y publicación del estudio, sin embargo, por el escaso argumento científico planteado, sus dificultades metodológicas, el conflicto de intereses y las conclusiones erróneas considero que debería ser reanalizado profundamente por el comité editorial y los lectores de la revista Colombiana de Obstetricia y Ginecología.

\section{José Enrique Sanín Blair}

Ginecología y Obstetricia; Medicina Materno

Fetal. Profesor titular

Universidad Pontificia Bolivariana

Medellín - Colombia

saninblair@yahoo.com

\section{REFERENCIAS}

1. Report of the National High Blood Pressure Education Program Working Group on High Blood Pressure in Pregnancy. Am J Obstet Gynecol 2000;183:S1-22.

2. Herrera JA. Nutritional factors and rest reduce pregnancy-induced hypertension and pre-eclampsia in positive roll-over test primigravidas. Int J Gynaecol Obstet 1993;41:31-5.

3. Herrera J, Areal-Herrera M, Herrera S. Prevention of preeclampsia by linoleic acid and calcium supplementation: a randomized controlled trial. Obstetric Gynecol 1998;91:585-90.

4. Herrera JA, Shahabuddin A, Faisal M, Ersheng G, Wei Y, Lixia D et al. Efectos de la suplementación oral con calcio y ácido linoleico conjugado en primigrávidas de alto riesgo. Colom Méd 2004;35:31-7.

5. Programa de Apoyo a la Reforma de Salud, Ministerio de la Protección Social. Guía de atención de las complicaciones hipertensivas asociadas con el embarazo. Guías de promoción de la salud y prevención de enfermedades en la salud pública. Tomo I. Bogotá DC: Ministerio de la Protección Social; 2007. p. 221-271.

6. Sarmiento Y, Crespo A, Portal ME, Breto AG, Menéndez YR. Morbilidad y mortalidad en neonatos hijos de madres toxémicas. Rev Cubana Pediatr 2009;81:10.

7. Rojas P, Pavón A, Rosso M, Losada A et al. Complicaciones a corto plazo de los recién nacidos pretérmino tardíos. An Pediatr 2011;75:169-74.

8. Vázquez JC, Vázquez J, Namfantche J. Asociación entre la hipertensión arterial durante el embarazo, bajo peso al nacer y algunos resultados del embarazo y el parto. Rev Cubana Obstet Ginecol [Internet] 2003;29.

9. Cami J. Conflicto de intereses e investigación clínica. Med Clin 1995; 105:174-9. 